\title{
Rigidity and Substitutive Dendric Words
}

\author{
V. Berthé \\ IRIF, CNRS UMR 8243, Université Paris Diderot-Paris 7, \\ Case 7014, 75205 Paris Cedex 13, France. \\ berthe@irif.fr \\ F. Dolce \\ LaCIM, Université du Québec à Montréal \\ 201, Président-Kennedy, H2X 3 Y7 Montréal (Québec), Canada. \\ francesco.dolce@lacim.ca \\ F. Durand \\ LAMFA, UMR CNRS 7352, Université de Picardie Jules Verne \\ 33, rue Saint-Leu, 80039 Amiens Cedex 1, France. \\ fabien.durand@u-picardie.fr \\ J. Leroy \\ Département de mathématiques, Université de Liège, \\ 12, Allée de la découverte, 4000 Liège, Belgium. \\ j.leroy@ulg.ac.be \\ D. Perrin \\ LIGM, UMR CNRS 8049, Université Paris-Est Marne-la-Vallée \\ 5, bd Descartes, Champs sur Marne 77454 Marne-la-Vallé Cedex 2, France. \\ dominique.perrin@esiee.fr \\ Received (Day Month Year) \\ Accepted (Day Month Year) \\ Communicated by (xxxxxxxxxx)
}

\begin{abstract}
Dendric words are infinite words that are defined in terms of extension graphs. These are bipartite graphs that describe the left and right extensions of factors. Dendric words are such that all their extension graphs are trees. They are also called tree words. This class of words includes classical families of words such as Sturmian words, codings of interval exchanges, or else, Arnoux-Rauzy words. We investigate here the properties of substitutive dendric words and prove some rigidity properties, that is, algebraic properties on the set of substitutions that fix a dendric word. We also prove that aperiodic minimal dendric subshifts (generated by dendric words) cannot have rational topological eigenvalues, and thus, cannot be generated by constant length substitutions.
\end{abstract}

Keywords: Dendric word; Rigidity; Substitution; S-adic word; Topological eigenvalue. 


\section{Introduction}

Dendric words are infinite words defined in terms of extension graphs that describe the left and right extensions of their factors. Extension graphs are bipartite graphs that can be roughly described as follows: given an infinite word $x$, and given a finite factor $w$ of $x$, one puts an edge between left and right copies of letters $a$ and $b$ such that $a w b$ is a factor of $x$. Dendric words are such that all their extension graphs are trees. For precise definitions, see Sections 2.1 and 2.2. This class of words with linear factor complexity includes classical families of words such as Sturmian words, codings of interval exchanges, or else, Arnoux-Rauzy words. Dendric words have striking combinatorial, ergodic and algebraic properties. This includes in particular algebraic properties of their return words [7], and of maximal bifix codes defined with respect to their languages $[2,8,9]$. They have been introduced in [7] and studied in several papers (as, for instance, $[8,9]$ ), under the name of tree words. We have chosen to call them here dendric words, and the subshifts they generate dendric subshifts, in order to avoid any ambiguity with respect to the notion of tree shift that refers to shifts defined on trees, and not on words (see e.g. [1]).

We investigate here the properties of substitutive dendric words and prove some rigidity properties. Rigidity has to do with the algebraic properties of the monoid of substitutions that fix a dendric word: an infinite word generated by a substitution is rigid if all the substitutions which fix this word are powers of a unique substitution. In the present paper, we concentrate on the iterative stabilizer according to the terminology of [25]: we focus on non-erasing morphisms and on infinite words generated by iterating a substitution.

There exist numerous results on the two-letter case concerning rigidity (see $[37,38]$ and also [4]). It is indeed well known that Sturmian words generated by substitutions are rigid $[37,38]$. The situation is more contrasted as soon as the size of the alphabet increases. For instance, over a ternary alphabet, the monoid of morphisms generating a given infinite word by iteration can be infinitely generated, even when the word is generated by iterating an invertible primitive morphism (see $[12,25])$.

Our main results are the following. We provide a characterization of substitutive primitive dendric words in terms of $\mathcal{S}$-adic expansions and tame substitutions (Theorem 7). An $\mathcal{S}$-adic expansion corresponds to the limit of compositions of substitutions of the form $\sigma_{1} \circ \cdots \circ \sigma_{n}$, and tame substitutions are elementary substitutions that extend to free group automorphisms (see Section 2.3 for definitions). We prove that if an infinite dendric word $x$ is fixed by two primitive substitutions $\sigma, \tau$, then these substitutions coincide up to powers, i.e., there exist positive integers $i, j$ such that $\sigma^{i}=\tau^{j}$; moreover, there exists a substitution $\theta$ such that all primitive substitutions of the stabilizer of $x$ are conjugate up to powers to $\theta$ (Theorem 9). We also prove that aperiodic minimal dendric subshifts cannot have rational topological eigenvalues (Theorem 13), and thus, they cannot be generated by constant length substitutions (Corollary 15). Our proofs rely on the notion of return words and on 
the so-called Return Theorem [7] that states that for every infinite dendric word defined over the alphabet $A$, the set of (right) return words is a basis of the free group generated by the alphabet $A$.

Let us briefly sketch the content of this paper. We recall in Section 2 the first basic definitions that are required, such as the notions of extension graphs and dendric words, return words, stabilizers, etc. We provide in Section 3 a characterization of substitutive dendric words in terms of derived sequences and $\mathcal{S}$-adic expansions. Rigidity properties are considered in Section 4. Lastly, we prove in Section 5 that recurrent dendric words cannot have rational topological eigenvalues. We conclude this paper with several questions in Section 6 .

\section{Basic definitions}

\subsection{Words, extensions and subshifts}

Let $A$ be a finite non-empty alphabet. All words considered below, unless stated explicitly, are supposed to be on the alphabet $A$. We denote by $\varepsilon$ the empty word of the free monoid $A^{*}$, by $A^{+}$the free semigroup and by $A^{\mathbb{N}}$ the set of infinite words over $A$. The Parikh vector of a word $w \in A^{*}$ is the vector in $\mathbb{N}^{A}$ whose coordinates are equal to the number of occurrences of letters in $w$, i.e., its $i$-th entry is equal to $|w|_{i}$, where $|w|_{i}$ stands for the number of occurrences of the letter $i$ in $w$. The notation $|w|$ stands for the length of $w$.

We say that a word $u$ is a factor of a word $w$ if there exist words $p, s$ such that $w=$ pus. If $p=\varepsilon$ (resp., $s=\varepsilon$ ) we say that $u$ is a prefix (resp., suffix) of $w$.

Let $F$ be a set of words on the alphabet $A$. For $w \in F$, we denote

$$
\begin{array}{lll}
L(w)=\{a \in A \mid a w \in F\}, & \ell(w)=\operatorname{Card}(L(w)), \\
R(w)=\{a \in A \mid w a \in F\}, & r(w)=\operatorname{Card}(R(w)), \\
B(w)=\{(a, b) \in A \times A \mid a w b \in F\}, & b(w)=\operatorname{Card}(B(w)) .
\end{array}
$$

Let $F$ be a set of words. For a word $w \in F$, we consider an undirected bipartite graph $\mathcal{E}(w)$ called its extension graph in $F$ and defined as follows: its set of vertices is the disjoint union of $L(w)$ and $R(w)$, and its edges are the pairs $(a, b) \in L(w) \times R(w)$ such that $(a, b) \in B(w)$. For an illustration, see Example 1 below.

Example 1. Let $F$ be a set of words on the alphabet $\{a, b\}$ having as factors of length less then 4 the set $\{\varepsilon, a, b, a a, a b, b a, a a b, a b a, b a a, b a b\}$. The extension graphs of the empty word and of the two letters are represented in Figure 1.

A set of words $F$ is factorial if it contains the factors of all its elements. It is biextendable if it is factorial and if for all $w \in F$, one has $r(w) \geq 1$ and $\ell(w) \geq 1$. It is recurrent if for every $u, v \in F$ there exists a word $w \in F$ such that $u w v \in F$. It is uniformly recurrent if it is biextendable and for any word $u \in F$, there exists an integer $n \geq 1$ such that $u$ is a factor of every word of $F$ of length $n$. Every uniformly recurrent set is recurrent while the opposite is in general not true. 


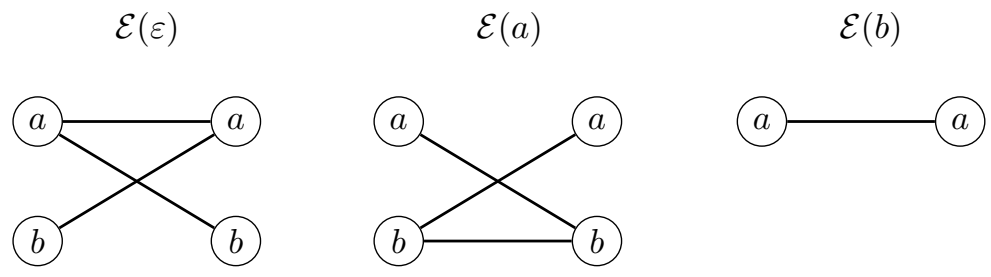

Fig. 1. The extension graphs of $\varepsilon$ (on the left), $a$ (on the center) and $b$ (on the right) are trees.

A word $w \in F$ is called right-special if $r(w) \geq 2$. It is called left-special if $\ell(w) \geq 2$. It is called bispecial if it is both right- and left-special. For a word $w \in F$, let

$$
m(w)=b(w)-\ell(w)-r(w)+1 .
$$

We say that $w$ is neutral (resp., weak, resp., strong) if $m(w)=0(\operatorname{resp} m(w)<0$, resp., $m(w)>0$ ). A factorial set $F$ is said to be neutral (resp., weak, resp., strong) if any word of $F$ is neutral (resp., neutral or weak, resp., neutral or strong). Note that our definition of neutral set corresponds to the one of neutral set of characteristic 1 in [14]. We will work here with a subclass of the family of neutral sets, namely dendric sets, introduced in Section 2.2. But before defining them, we also introduce notions corresponding to infinite words and subshifts.

An infinite word in $A^{\mathbb{N}}$ is said to be uniformly recurrent if the set of its factors is uniformly recurrent. In other words, an infinite word $x=\left(x_{n}\right)_{n \in \mathbb{N}}=x_{0} x_{1} \ldots$ is uniformly recurrent if every word occurring in $x$ occurs in an infinite number of positions with bounded gaps, that is, if for every factor $w$, there exists a positive integer $s$ such that for every $n, w$ is a factor of $x_{n} \ldots x_{n+s-1}$. The set of factors $F(x)$ of an infinite word $x$ is called its language.

The mapping $S$ acting on sets of infinite words is the (one-sided) shift $S$ acting on $A^{\mathbb{N}}$ :

$$
S\left(\left(x_{n}\right)_{n \in \mathbb{N}}\right)=\left(x_{n+1}\right)_{n \in \mathbb{N}} .
$$

A subshift is a pair $(X, S)$ where $X$ is a closed shift-invariant subset of some $A^{\mathbb{N}}$. A subshift is said to be minimal if it admits no non-trivial closed and shift-invariant subset. A subshift $X$ is minimal if and only if every infinite word $x \in X$ is uniformly recurrent. If $X$ is a subshift, then its language $F(X)$ is defined as the set of factors of elements of $X$. Given a biextendable set of words $F \subset A^{*}$, there exists a unique subshift $X$ such that $F(X)=F$. We say that a minimal subshift is periodic whenever it is finite. Otherwise it is said to be aperiodic.

\subsection{Dendric sets}

The following notion has been introduced in [7], under the terminology of tree set. We say that a set of words $F$ is a dendric set if it is biextendable and if for every 
word $w \in F$, the graph $\mathcal{E}(w)$ is a tree. Note that a biextendable set $F$ is a dendric set if and only if the graph $\mathcal{E}(w)$ is a tree for every bispecial word $w$. Indeed the extension graph associated with every non-bispecial word is trivially a tree.

If the extension graph $\mathcal{E}(w)$ of $w$ is a tree, then $m(w)=0$. Thus $w$ is neutral. Note that if $\mathcal{E}(w)$ is acyclic, one has $m(w)=1-c$, where $c$ is the number of connected components of the graph $\mathcal{E}(w)$. Sturmian sets and regular interval exchange sets are examples of dendric sets (see [7]).

The sequence $\left(p_{n}\right)_{n \geq 0}$ with $p_{n}=\operatorname{Card}\left(F \cap A^{n}\right)$ is called the factor complexity of $F$. Set $k=\operatorname{Card}(F \cap A)-1$. The factor complexity of a neutral set $F$ is equal to $k n+1$ (see [7]). Since a dendric set is neutral, we deduce that the factor complexity of a dendric set is also $k n+1$ (see [7]).

The following result shows that in neutral sets (and thus in dendric sets) the notion of recurrence and uniformly recurrence coincide.

Proposition 2 (Corollary 5.3 [14]) A recurrent neutral set is uniformly recurrent.

We similarly define a dendric word as an infinite word $x$ such that its language $F(x)$ is a dendric set, and a dendric subshift as a subshift $(X, S)$ such that $F(X)$ is a dendric set.

\subsection{Matrices and free groups}

A morphism $\sigma: A^{*} \rightarrow B^{*}$ is a monoid morphism from $A^{*}$ into $B^{*}$. We consider here exclusively non-erasing morphisms, that is, morphisms such that the image of every element in $A^{+}$belongs to $B^{+}$. When $B=A$, such a morphism is a substitution. If there exists a letter $a \in A$ such that the word $\sigma(a)$ begins with $a$ and if $\left|\sigma^{n}(a)\right|$ tends to infinity with $n$, there exists a unique infinite word denoted $\sigma^{\omega}(a)$ which has all words $\sigma^{n}(a)$ as prefixes. Such an infinite word is called a fixed point of the substitution $\sigma$.

A substitution $\sigma: A^{*} \rightarrow A^{*}$ is called primitive if there is a positive integer $k$ such that for all $a, b \in A$, the letter $b$ appears in $\sigma^{k}(a)$. If $\sigma$ is a primitive substitution, there exists a power $\sigma^{k}$ that admits a fixed point, and the set of factors of any fixed point of $\sigma$ (or of some power of $\sigma$ ) is uniformly recurrent (see for example Proposition 1.2.3 in [22]). Furthermore, all these fixed points have the same language that we call the language of the substitution.

An infinite word $x$ over the alphabet $A$ is said to be substitutive if there exist a substitution $\sigma$ over an alphabet $B$ with a fixed point $y=\sigma^{\omega}(b)$, for some $b \in B$, and a morphism $\tau: B^{*} \rightarrow A^{*}$, such that $\tau(y)=x$. It is said substitutive primitive when $\sigma$ is primitive.

The incidence matrix (also called substitution matrix) of a substitution $\sigma$ defined over the alphabet $A$ is the $A \times A$-matrix whose entry $(i, j)$ counts the number $|\sigma(j)|_{i}$ of occurrences of the letter $i$ in $\sigma(j)$.

The subshift $\left(X_{\sigma}, S\right)$ generated by a primitive substitution $\sigma$ over $A$ is the set 
of infinite words $x$ such that any word $w$ in the language $F(x)$ is a factor of some $\sigma^{n}(a)$, for some $a \in A$ and some positive integer $n$. Such a subshift is minimal.

Example 3. Let $\sigma_{F}$ be the Fibonacci morphism defined over the alphabet $\{a, b\}$ by $\sigma_{F}(a)=a b$ and $\sigma_{F}(b)=a$. The morphism $\sigma_{F}$ is a primitive subtistitution and the uniformly recurrent infinite word $\sigma_{F}^{\omega}(a)=$ abaababaabaab $\cdots$ is called the Fibonacci word. The incidence matrix of $\sigma_{F}$ is

$$
\left(\begin{array}{ll}
1 & 1 \\
1 & 0
\end{array}\right)
$$

The set of factors of the Fibonacci word, called the Fibonacci set, is a dendric set (see, e.g., [7]) and the extensions graphs of $\varepsilon, a$ and $b$ are shown in Figure 1.

We denote by $\mathbb{F}_{A}$ the free group on the alphabet $A$. It is the set of all words on the alphabet $A \cup A^{-1}$ which are reduced, in the sense that they do not have any factor $a a^{-1}$ or $a^{-1} a$ for $a \in A$. A morphism $\sigma$ from $A^{*}$ to $A^{*}$ can be extended to a morphism from $\mathbb{F}_{A}$ to $\mathbb{F}_{A}$ by defining $\sigma\left(a^{-1}\right)=(\sigma(a))^{-1}$ for all $a \in A \cup A^{-1}$. A morphism $\sigma$ of the free monoid is said to be invertible if, when extended to a morphism of the free group it is an automorphism, that is, there exists a morphism $\tau$ from $\mathbb{F}_{A}$ to $\mathbb{F}_{A}$ such that $\sigma \circ \tau=\tau \circ \sigma=$ Id. In particular, if $\sigma$ is such that there exists $n$ such that $\sigma^{n}$ is an automorphism, then $\sigma$ is itself an automorphism.

An automorphism $\alpha$ of the free group on $A$ is positive if $\alpha(a)$ belongs to $A^{+}$ for every $a \in A$. We say that a positive automorphism of the free group on $A$ is tame if it belongs to the submonoid generated by the permutations of $A$ and the automorphisms $\alpha_{a, b}, \tilde{\alpha}_{a, b}$ defined for $a, b \in A$, with $a \neq b$, by

$$
\alpha_{a, b}(c)=\left\{\begin{array}{ll}
a b & \text { if } c=a, \\
c & \text { otherwise }
\end{array} \quad \text { and } \quad \tilde{\alpha}_{a, b}(c)= \begin{cases}b a & \text { if } c=a, \\
c & \text { otherwise. }\end{cases}\right.
$$

Thus $\alpha_{a, b}$ places a letter $b$ after each $a$ and $\tilde{\alpha}_{a, b}$ places a letter $b$ before each $a$, without modifying the other letters. The above automorphisms and the permutations of $A$ are called the elementary positive automorphisms on $A$. We let $\mathcal{S}_{e}$ denote the set of elementary positive automorphisms. This is a subset of the set of Nielsen's transformations (see, e.g., [30]). A substitution that extends as a positive automorphism that is tame is said to be a tame substitution.

The monoid of tame substitutions strictly contains the monoid of episturmian morphisms, also called Arnoux-Rauzy substitutions. Note that the case of a twoletter alphabet corresponds to the Stumian case. The monoid of episturmian morphisms has been thoroughly investigated, e.g., in [36]. It is generated by the permutations together with the set of automorphisms $\psi_{a}, \tilde{\psi}_{a}$, defined for $a \in A$ by

$$
\psi_{a}(c)=\left\{\begin{array}{ll}
a c & \text { if } c \neq a, \\
a & \text { if } c=a .
\end{array} \text { and } \quad \tilde{\psi}_{a}(c)= \begin{cases}c a & \text { if } c \neq a, \\
a & \text { if } c=a .\end{cases}\right.
$$


The submonoid of the monoid of episturmian morphisms generated by the permutations together with the set of automorphisms $\psi_{a}$, defined for $a \in A$ (that is, no $\tilde{\psi}_{b}$ is allowed) is called the monoid of epistandard morphisms.

Note also that the monoid of tame automorphisms is strictly included in the monoid of positive automorphisms. This is a consequence of the fact that the monoid of positive automorphisms on an alphabet containing at least three letters is not finitely generated $[36,39,41]$. However, invertible substitutions over a binary alphabet are exactly the Sturmian substitutions (see, e.g., [31,40]), and the monoid of all invertible substitutions (i.e., the Sturmian monoid) is finitely generated.

Tame substitutions are closely related to dendric words such as shown in Section 3 where a characterization of substitutive dendric words is provided. But, not every tame substitution admits as a fixed point a dendric word (see Example 5.25 in [8]). The situation is thus more contrasted than in the Sturmian case where every Sturmian substitution generates a Sturmian word (see, e.g., [29]). Note however that it is decidable whether the language of a primitive aperiodic substitution ${ }^{\mathrm{a}}$ is a dendric set (see [13]).

\subsection{Stabilizers}

The stabilizer of an infinite word $x \in A^{\mathbb{N}}$, denoted by $\operatorname{Stab}(x)$, is the monoid of substitutions $\sigma$ defined on the alphabet $A$ that satisfy $\sigma(x)=x$. Words that have a cyclic stabilizer are called rigid ${ }^{\mathrm{b}}$.

Note that we concentrate here on the iterative stabilizer according to the terminology of [25]. Results on the possible growth of elements of the stabilizer are provided in [12] and [20]. It is shown in particular that polynomial and exponential growth cannot co-exist in the stabilizer for aperiodic words.

Words generated by Sturmian substitutions are rigid (see [38]). It is proved in [25] that Arnoux-Rauzy words that are fixed points of epistandard morphisms are rigid.

Note also that the question of the existence of non-negative integers $n, p$ such that $\sigma^{n}=\tau^{p}$ is decidable [35].

\subsection{Return words}

Let $F \subset A^{*}$ be a set of words. For $w \in F$, let

$$
\Gamma_{F}(w)=\left\{x \in F \mid w x \in F \cap A^{+} w\right\} \quad \text { and } \quad \Gamma_{F}^{\prime}(w)=\left\{x \in F \mid x w \in F \cap w A^{+}\right\} .
$$

If $F$ is recurrent, the sets $\Gamma_{F}(w)$ and $\Gamma_{F}^{\prime}(w)$ are non-empty. Let

$$
\mathcal{R}_{F}(w)=\Gamma_{F}(w) \backslash \Gamma_{F}(w) A^{+} \quad \text { and } \quad \mathcal{R}_{F}^{\prime}(w)=\Gamma_{F}^{\prime}(w) \backslash A^{+} \Gamma_{F}^{\prime}(w)
$$

\footnotetext{
${ }^{a}$ A primitive substitution is said to be aperiodic if the subshift it generates is aperiodic.

bote that rigidity has nothing to do with the ergodic notion of rigidity.
} 
be respectively the set of right return words and the set of left return words to $w$. In other words, a right return word to $w$ is a word $x$ such that $w x$ is in $F, w$ is a suffix of $w x$ and $w x$ contains exactly two occurrences of $w$, and a left return word to $w$ is a word $x$ such that $x w$ is in $F, w$ is a prefix of $x w$ and $x w$ contains exactly two occurrences of $w$. Note that $w \mathcal{R}_{F}(w)=\mathcal{R}_{F}^{\prime}(w) w$. Note also that a recurrent set $S$ is uniformly recurrent if and only if the set $\mathcal{R}_{S}(w)$ is finite for any $w \in S$.

Return words will play a crucial role in the following. The following theorem is proved in [7] and it is referred as the Return Theorem.

Theorem 4 (Theorem $4.5[7]$ ) Let $F$ be a recurrent dendric set containing the alphabet $A$. Then, for any $w \in S$, the set $\mathcal{R}_{F}(w)$ is a basis of the free group $\mathbb{F}_{A}$ on A. Similarly, for any $w \in S$, the set $\mathcal{R}_{F}^{\prime}(w)$ is a basis of the free group on $A$.

\section{Substitutive dendric words}

We first recall some basic definitions concerning $\mathcal{S}$-adic representations in terms of return words. For more on $\mathcal{S}$-adic words see, e.g., [3, 21, 26, 27, 28].

An infinite word $x \in A^{\mathbb{N}}$ is said to be $\mathcal{S}$-adic if there is a sequence of alphabets $\left(A_{n}\right)_{n \in \mathbb{N}}$, a sequence of morphisms $\mathbf{s}=\left(\sigma_{n}: A_{n+1}^{*} \rightarrow A_{n}^{*}\right)_{n \in \mathbb{N}}$ and a sequence of letters $\mathbf{a}=\left(a_{n} \in A_{n}\right)_{n \in \mathbb{N}}$ such that $x=\lim _{n \rightarrow+\infty} \sigma_{0} \sigma_{1} \cdots \sigma_{n}\left(a_{n+1}\right)$. The set $\mathcal{S}$ in the terminology $\mathcal{S}$-adic refers to the set of morphisms $\mathcal{S}=\left\{\sigma_{n} \mid n \in \mathbb{N}\right\}$. The pair $(\mathbf{s}, \mathbf{a})$ is called an $\mathcal{S}$-adic representation of $x$ and the sequence $\mathbf{s}$ a directive sequence of $x$. The pair (s, a) is eventually periodic if there exists a non-negative integer $N$ and a positive integer $n$ such that $\left(\sigma_{m+n}, a_{m+n}\right)=\left(\sigma_{m}, a_{m}\right)$ for all $m \geq N$, and it is purely periodic if there exists a positive integer $n$ such that $\left(\sigma_{m+n}, a_{m+n}\right)=\left(\sigma_{m}, a_{m}\right)$ for all $m \in \mathbb{N}$. The pair $(\mathbf{s}, \mathbf{a})$ is said to be primitive whenever the directive sequence s is primitive, i.e., for all $r \geq 0$, there exists $r^{\prime}>r$ such that all letters of $A_{r}$ occur in $\sigma_{r} \sigma_{r+1} \cdots \sigma_{r^{\prime}}(a)$ for all $a \in A_{r^{\prime}+1}$. Observe that if $x$ has a primitive $\mathcal{S}$-adic representation, then $x$ is uniformly recurrent (see, e.g., [17]). If $X$ is a minimal subshift and if $x \in X$ admits a primitive $\mathcal{S}$-adic representation (s,a), then we say that $(\mathbf{s}, \mathbf{a})$ is also an $\mathcal{S}$-adic representation of $X$.

Return words provide $\mathcal{S}$-adic representations of uniformly recurrent words. Indeed, let $x=\left(x_{n}\right)_{n \in \mathbb{N}} \in A^{\mathbb{N}}$ be a uniformly recurrent word. We consider a factorization in left return words with respect to the first letter $x_{0}$ of $x$ (note that such return words starts with $x_{0}$ ). Denoting by $F$ the language of $x$, there exists a unique sequence $\left(w_{n}\right)_{n \in \mathbb{N}} \in\left(\mathcal{R}_{F}^{\prime}\left(x_{0}\right)\right)^{\mathbb{N}}$ such that $x=w_{0} w_{1} w_{2} \cdots$. Let $R$ be the alphabet $\left\{1,2, \ldots, \operatorname{Card}\left(\mathcal{R}_{F}^{\prime}\left(x_{0}\right)\right)\right\}$ and consider the return morphism $\lambda: R^{*} \rightarrow A^{*}$ such that $\lambda(R)=\mathcal{R}_{F}^{\prime}\left(x_{0}\right)$ and $\lambda(i)$ is the $i$-th return word occurring in $\left(w_{n}\right)_{n \in \mathbb{N}}$ for all $i$. More precisely, $\lambda$ is such that for all $i \in R$ and all $m \in \mathbb{N}$, if $w_{m} \notin\left\{w_{0}, w_{1}, \cdots, w_{m-1}\right\}=\{\lambda(j) \mid 1 \leq j<i\}$, then $\lambda(i)=w_{m}$. The derived sequence of $x$ is the unique sequence $\mathcal{D}(x) \in R^{\mathbb{N}}$ such that $\lambda(\mathcal{D}(x))=x$. We recursively define $\mathcal{D}^{n}(x), \lambda_{n}$ and $R_{n}$ by $\mathcal{D}^{0}(x)=x, R_{0}=A$ and $\mathcal{D}^{n}(x)=\mathcal{D}\left(\mathcal{D}^{n-1}(x)\right) \in R_{n}^{\mathbb{N}}$ with $\lambda_{n}: R_{n+1}^{*} \rightarrow R_{n}^{*}$ the return morphism of $\mathcal{D}^{n}(x)$. 
Let us consider the set of morphisms $\Lambda=\left\{\lambda_{n} \mid n \in \mathbb{N}\right\}$. We denote $\lambda=$ $\left(\lambda_{n}\right)_{n \in \mathbb{N}}$ and $\mathbf{1}=(1)_{n \geq 1}$ the constant sequence that takes the value 1 . The pair $(\lambda, \mathbf{1})$ is clearly primitive (see also Proposition 5.22 in [8]). We call it the $\Lambda$-adic representation of $x$. For all $n \geq 1$, we set $\theta_{n}=\lambda_{0} \lambda_{1} \cdots \lambda_{n-1}: R_{n}^{*} \rightarrow A^{*}$. Thus we have $\theta_{n}\left(\mathcal{D}^{n}(x)\right)=x$.

The $\Lambda$-adic representation allows the following formulation for characterizing primitive substitutive words.

Theorem 5 ([16]) A uniformly recurrent word $x \in A^{\mathbb{N}}$ is primitive substitutive if and only if the set of its derived sequence $\left\{\mathcal{D}^{n}(x) \mid n \in \mathbb{N}\right\}$ is finite.

The next result implies that any recurrent dendric word admits a primitive $\mathcal{S}_{e^{-}}$ adic representation (see also Theorem 5.23 in [8]). The main property used below is that in a recurrent dendric set the set of return words of a given word forms a basis of the free group (Theorem 4.5 in [7]). We recall that $\mathcal{S}_{e}$ stands for the set of elementary positive automorphisms such as defined in Section 2.3 .

Theorem 6. Let $x \in A^{\mathbb{N}}$ be a recurrent dendric word over the alphabet $A=$ $\{1, \ldots, d\}$ and let $(\lambda, \mathbf{1})$ be its $\Lambda$-adic representation in return words.

(1) For all $n \in \mathbb{N}, \mathcal{D}^{n}(x) \in A^{\mathbb{N}}$ is a recurrent dendric word.

(2) For all $n \in \mathbb{N}$, the morphism $\lambda_{n}: A^{*} \rightarrow A^{*}$ extends to a tame automorphism of $\mathbb{F}_{A}$.

In other words, the $\Lambda$-adic representation of $x$ provides an $\mathcal{S}_{e}$-adic representation of $x$.

Proof. By Proposition 2, $x$ is uniformly recurrent. Item 1 then follows from Theorem 5.13 in [8]. Item 2 follows from Theorem 4.5 in [7] and Theorem 5.19 in [8]. $\square$

We now can state the main result of this section that provides a characterization of primitive substitutive dendric words. A similar statement is known to hold for Sturmian words (see, e.g., [5]). In the latter case it can even be expressed in terms of eventually periodic continued fractions and Ostrowski expansions.

Theorem 7. A recurrent dendric word is primitive substitutive if and only if it has an eventually periodic primitive $\mathcal{S}_{e}$-adic representation.

Proof. One easily checks that the condition is sufficient. The necessary part follows from Theorem 5: the set $\left\{\mathcal{D}^{n}(x) \mid n \in \mathbb{N}\right\}$ being finite, there exists $m, n \in \mathbb{N}, m<n$ such that $\mathcal{D}^{m}(x)=\mathcal{D}^{n}(x)$. By construction of $\lambda$, this means that for all $k \in \mathbb{N}$, $\mathcal{D}^{m+k}(x)=\mathcal{D}^{n+k}(x)$ and $\lambda_{m+k}=\lambda_{n+k}$, i.e., $(\lambda, \mathbf{1})$ is eventually periodic. We then apply Theorem 6 .

Observe that if the $\Lambda$-adic representation of a uniformly recurrent dendric word $x$ is purely periodic, then $x$ is the fixed point of a primitive tame substitution. The converse is not true, such as illustrated by the following example. 
Example 8. Let us consider the primitive substitution

$$
\sigma: 0 \mapsto 010, \quad 1 \mapsto 10
$$

and its fixed point $x=\sigma^{\omega}(0)$. One has $x=\lambda_{0} \lambda_{1}\left(\lambda_{2}\right)^{\omega}(0)$ where

$$
\lambda_{0}:\left\{\begin{array}{l}
1 \mapsto 01 \\
2 \mapsto 0
\end{array}, \quad \lambda_{1}:\left\{\begin{array}{l}
1 \mapsto 1 \\
2 \mapsto 12
\end{array}, \quad \text { and } \quad \lambda_{2}:\left\{\begin{array}{l}
1 \mapsto 12 \\
2 \mapsto 122
\end{array} \quad\right.\right.\right.
$$

Thus, the $\Lambda$-adic representation of $x$ is eventually periodic, but not purely periodic. Let $\pi: 1 \mapsto 1,2 \mapsto 0$. Note that $\lambda_{0}=\pi \circ \tilde{\alpha}_{1,2}, \lambda_{1}=\tilde{\alpha}_{2,1}, \lambda_{2}=\alpha_{1,2} \tilde{\alpha}_{2,1}$.

\section{Stabilizers of dendric words}

The following theorem states that one has a weak form of rigidity for dendric words together with a structure theorem for the stabilizer of a dendric word. Recall that recurrent dendric words are uniformly recurrent by Proposition 2.

Theorem 9. Let $x$ be a dendric word. Primitive substitutions in the stabilizer Stab $(x)$ of $x$ coincide up to powers. More precisely, if $x$ is a fixed point of both $\sigma$ and $\tau$ primitive substitutions, then there exist $i, j \geq 1$ such that $\tau^{i}=\sigma^{j}$.

Let $x$ be a recurrent substitutive dendric word. There is a primitive tame substitution $\theta$ such that any primitive substitution $\sigma \in \operatorname{Stab}(x)$ has a power that is (tamely) conjugate to a power of $\theta$, that is, there exists a positive tame automorphism $\tau$ such that $\sigma^{i}=\tau \theta^{j} \tau^{-1}$, for some $i, j \geq 1$.

In particular, if $x$ is a dendric word, any primitive substitution in Stab $(x)$ extends to an automorphism of the free group and is a tame substitution.

Note that the first statement implies that the Perron-Frobenius eigenvalues of $\sigma$ and $\tau$ are multiplicatively dependent, which is also a consequence of Cobham's Theorem [18].

Proof. The first statement is a direct consequence of Corollary 22 in [19]. Indeed, given any finite word $w$, the set of Parikh vectors of returns words to $w$ generates $\mathbb{Z}^{d}$ by the Return Theorem (see Theorem 4 ).

Now, let $x$ be a recurrent dendric word. If $x$ is primitive substitutive, then the set $\left\{\mathcal{D}^{n}(x) \mid n \in \mathbb{N}\right\}$ is finite by Theorem 5 . Let $(\lambda, \mathbf{1})$ be its $\Lambda$-adic representation, and, $k, l \in \mathbb{N}, k<l$, be such that $\mathcal{D}^{k}(x)=\mathcal{D}^{l}(x)$ and all derived sequences in $\left\{\mathcal{D}^{n}(x) \mid 0 \leq n<l\right\}$ are pairwise distinct. Let $\theta$ be the morphism such that $\theta_{l}=\theta_{k} \theta$, i.e., $\theta=\lambda_{k} \lambda_{k+1} \cdots \lambda_{l-1}$. Since $\left(\left(\lambda_{n}\right)_{n \geq k}, \mathbf{1}\right)$ is a primitive $\Lambda$-adic representation of $\mathcal{D}^{k}(x), \theta$ is a primitive substitution having $\mathcal{D}^{k}(x)$ as a fixed point.

Let $\sigma$ be a primitive substitution in $\operatorname{Stab}(x)$. There exists a primitive substitution $\sigma_{k}$ satisfying $\sigma \theta_{k}=\theta_{k} \sigma_{k}$ and having $\mathcal{D}^{k}(x)$ for fixed point, by Proposition 5.1 in [16]. By Theorem 6 and by the first statement, we have $\sigma_{k}^{i}=\theta^{j}$ for some $i, j \geq 1$. We thus get $\sigma^{i}=\theta_{k} \theta^{j} \theta_{k}^{-1}$, which finishes the proof of the second statement.

We deduce that if $\sigma$ is a primitive element of $\operatorname{Stab}(x)$, then it is invertible. By Theorem 5.19 in [8], it is thus a tame substitution. 


\section{Dendric subshifts and topological eigenvalues}

We prove in this section that aperiodic minimal dendric subshifts cannot be generated by substitutions of constant length (see Corollary 15 below). We provide here a spectral proof and prove the more general result that aperiodic minimal dendric subshifts cannot have rational eigenvalues. Let us start by recalling some definitions.

Let $(X, S)$ be a subshift. We say that $(X, S)$ is totally minimal whenever $\left(X, S^{n}\right)$ is minimal for all $n$.

A cyclic partition of $(X, S)$ is a partition $X=\cup_{i=1}^{m} X_{i}$ in closed subsets such that $X_{i+1}=S\left(X_{i}\right)$, for $1 \leq i \leq m-1$, and $S\left(X_{m}\right)=X_{1}$. Note that the elements $X_{i}$ are thus clopen sets.

A topological eigenvalue of $(X, S)$ is a complex number $\lambda$ such that there exists a non-zero continuous function $f: X \rightarrow \mathbb{C}$ satisfying $f \circ S=\lambda f$. The function $f$ is called a topological eigenfunction associated with $\lambda$. A topological eigenvalue of the form $\exp (2 i \pi k / n)$, for some $n \geq 2$ and some integer $k$, is said to be a rational topological eigenvalue.

Example 10. Let $\sigma_{T M}$ be the Thue-Morse substitution defined on $\{a, b\}^{*}$ by $\sigma_{T M}(a)=a b$, and $\sigma_{T M}(b)=b a$. Let $X_{1}=\sigma_{T M}[a] \cup \sigma_{T M}[b]$.

The partition $\left(X_{1}, S X_{1}\right)$ is a cyclic partition of the subshift $X_{T M}$ generated by $\sigma_{T M}$. Indeed, one checks that $S^{2} X_{1}=X_{1}$, and moreover that $X_{1}$ and $S X_{1}$ are disjoint, by recognizability of the Thue-Morse substitution [32,33].

The eigenvalue $-1=\exp (i \pi)$ is a topological eigenvalue. Indeed, consider the function $f$ that maps every element of $X_{1}$ to the constant value -1 , and every element of $S X_{1}$ to the constant value 1 . One has $f(S x)=-f(x)$ for every element $x$ of $X_{T M}$.

The following result is part of the folklore of topological dynamical systems (see, e.g., [34]). It shows that these notions are intimately related.

Lemma 11. Let $(X, S)$ be a minimal subshift. The following are equivalent.

(1) $(X, S)$ has a cyclic partition $X_{1}, \ldots, X_{n}$ for some $n \geq 2$;

(2) $\exp (2 i \pi / n)$ is a topological eigenvalue of $(X, S)$ for some $n \geq 2$;

(3) $(X, S)$ is not totally minimal.

Proof. It is clear that (3) is equivalent to (1).

Let us prove that (1) implies (2). Let $f$ be defined as the constant function taking the value $\exp (2 i \pi k / n)$ on $X_{k}$. One checks that $f$ is a topological eigenfunction associated with the eigenvalue $\exp (2 i \pi / n)$.

Conversely, let us prove that (2) implies (1). Let $\exp (2 i \pi / n)$ be a topological eigenvalue of $(X, S)$, and let $f$ be a topological eigenfunction for this eigenvalue. One can suppose $f(x)=1$ for some $x \in X$. One has $f\left(S^{n} x\right)=f(x)=1$. By minimality, every element $y$ of $X$ can be written as $y=\lim S^{n_{i}}(x)$, for some nondecreasing subsequence $\left(n_{i}\right)_{i}$. There exists $k$ with $0 \leq k \leq n-1$ such that in- 
finitely many $n_{i}$ are congruent to $k$ modulo $n$. By continuity of $f$, one has $f(y)=$ $\exp (2 i \pi k / n) f(x)=\exp (2 i \pi k / n)$. Hence $f(X)=\{\exp (2 i \pi k / n) \mid 0 \leq k \leq n-1\}$. Let $X_{k}=f^{-1}(\{\exp (2 i \pi k / n)\})$, for $0 \leq k \leq n-1$. This defines a cyclic partition. $\square$

We now prove that minimal dendric subshifts are totally minimal. We use below the fact that the set of factors of a given length over the alphabet $A$ is a code, as well as the properties of stability of dendric sets by maximal bifix decoding, and the fact that recurrent dendric sets are in fact uniformly recurrent. Recall that a set $G \subset A^{+}$of non-empty words over the alphabet $A$ is a code if the relation

$$
g_{1} \cdots g_{n}=h_{1} \cdots h_{m}
$$

with $n, m \geq 1$ and $g_{1}, \ldots, g_{n}, h_{1}, \ldots, h_{m} \in G$ implies $n=m$ and $g_{i}=h_{i}$ for $i=1, \ldots, n$. A coding morphism for a code $G \subset A^{+}$is a morphism $f: B^{*} \rightarrow A^{*}$ which maps bijectively $B$ onto $G$.

In the next proposition we use the fact that the set of factors of a fixed length of a minimal subshift $X$ is a $F(X)$-maximal bifix code (see [8]).

Proposition 12. A minimal dendric subshift is totally minimal.

Proof. Let $(X, S)$ be a minimal dendric subshift. Let $n \geq 2$. We consider the code $G$ made of the factors of the language of $X$ of length $n$, that is, $G=F(X) \cap A^{n}$. Let $f: B \rightarrow G$ be a coding morphism for $G$. Let $H=f^{-1}(F(X))$ and let $(Y, T)$ be the subshift defined by $F(Y)=H$, with $T$ standing for the shift acting on $Y$. One thus has $Y \subset B^{\mathbb{N}}$, with $B$ being in bijection with the set $G$ of factors of length $n$ of $X$. Then $f$ extends to an isomorphism from $(Y, S)$ onto $\left(X, S^{n}\right)$. Since $G$ is an $F(X)$ maximal bifix code, the set $H$ is a recurrent dendric set by [8]. By Proposition 2 (i.e., by Corollary 5.3 in [14]) this implies that $(Y, T)$ is minimal, which yields that $\left(X, S^{n}\right)$ is minimal.

We now can state the main result of this section.

Theorem 13. Let $(X, S)$ be an aperiodic minimal dendric subshift. Then it admits no rational topological eigenvalue.

Proof. This is a direct consequence of Proposition 12 together with Lemma 11.

As a consequence, we deduce that Arnoux-Rauzy subshifts and regular interval exchanges cannot have rational topological eigenvalues. For examples of interval exchanges having topological eigenvalue, see, e.g., [23], and for examples of ArnouxRauzy subshifts having topological eigenvalue, see [10].

Example 14. Consider the Tribonacci substitution $\sigma_{T}$ defined over the alphabet $\{a, b, c\}$ by $\sigma_{T}(a)=a b, \sigma_{T}(b)=a c$ and $\sigma_{T}(c)=a$. Let $X_{T}$ be the subshift generated by $\sigma_{T}$. The subshift $X_{T}$ is an aperiodic dendric subshift by [2]. It thus admits no rational topological eigenvalue. 
Let $\tau$ be the substitution defined over the alphabet $\{a, b, c\}$ by $\tau(a)=a b, \tau(b)=a c$ and $\tau(c)=a a$. Let $X_{\tau}$ be the subshift generated by $\tau$. Let $X_{1}=\tau[a] \cup \tau[b] \cup \tau[c]$. The partition $\left(X_{1}, S X_{1}\right)$ is a cyclic partition of $X_{\tau}$. Indeed, one checks, as in Example 10, that $S^{2} X_{1}=X_{1}$, and moreover that $X_{1}$ and $S X_{1}$ are disjoint, by recognizability of this primitive substitution [32,33]. The subshift $(X, S)$ admits -1 as a rational topological eigenvalue. Thus, the subshift $(X, S)$ is not a dendric subshift.

We recall that a subshift generated by a primitive constant length substitution admits rational topological eigenvalues (see, e.g., [11]). A subshift $X$ is said to be Toeplitz if any element $x=\left(x_{n}\right)_{n} \in X$ satisfies the following: for all $n$, there exists a positive integer $p$ such that $x_{n}=x_{n+k p}$, for all $k$. For more on Toeplitz subshifts, see, e.g., [15]. Toeplitz subshifts are also known to have rational topological eigenvalues.

Corollary 15. Let $(X, S)$ be an aperiodic minimal dendric subshift. Then, it can neither be generated by a primitive constant length substitution, nor be a Toeplitz subshift.

\section{Concluding questions}

Let us recall that Arnoux-Rauzy words that are fixed points of epistandard morphisms are rigid, as proved in [25]. We are not able here to answer the question asked in [25] on the rigidity of Arnoux-Rauzy words that are fixed points of episturmian morphisms (that not necessarily epistandard ones), even if Theorem 9 provides some elements of answer. Nevertheless, we extend this question to the general framework of dendric words. We then ask the following: are recurrent dendric words or minimal dendric subshifts rigid?

By analogy with the monoid of epistandard morphisms, we introduce the monoid of standard tame substitutions as the monoid generated by the permutations of $A$ and the automorphisms $\alpha_{a, b}$, for $a, b \in A$ with $a \neq b$, and the monoid of antistandard tame substitutions as the monoid generated by the permutations of $A$ and the automorphisms $\tilde{\alpha}_{a, b}$, for $a, b \in A$ with $a \neq b$. There is a simple characterization of elements of the monoid of standard tame substitutions which extends Lemma 2.4 in [36]: a tame substitution is standard (resp., antistandard) if and only if the set of the first (resp., last) letters of the images of letters in $A$ is equal to $A$. However, one notable difference with the episturmian case is that the tame automorphism $\alpha_{a, b}$ is not rotationally conjugate to $\widetilde{\alpha}_{a, b}$, whereas $\widetilde{\psi}_{a}$ is rotationally conjugate to $\psi_{a}$. We recall that two substitutions $\sigma$ and $\rho$ over the finite alphabet $A$ are said to be rotationally conjugate if $\sigma=\gamma_{w} \circ \rho$ for some $w \in A^{*}$, where $\gamma_{w}$ is the inner automorphism of $\mathbb{F}_{A}$ defined by $\gamma_{w}(x)=w x w^{-1}$, for all $x \in \mathbb{F}_{A}$. Another difference comes from the fact that not every fixed point of a tame substitution is a dendric word (see Example 5.25 in [8]).

Several questions occur naturally. Do fixed points of standard tame substitutions play a role analogous for dendric words as the role played by fixed points of epistandard morphisms for Arnoux-Rauzy words, in particular with respect to special 
factors? Is this notion relevant in the present context? What can be said on tame substitutions that have the same incidence matrix? What can be said when one exchanges $\widetilde{\alpha}_{a, b}$ with $\alpha_{a, b}$ in a decomposition of a tame substitution? Can rotational conjugacy be seen on the decomposition by elementary morphisms of a substitution that preserves a dendric word?

Let $x$ be a recurrent dendric word. If $x$ is a fixed point of a substitution, is this substitution primitive? In other words, is any non-trivial element of Stab $(x)$ primitive? Moreover, can one characterize in terms of the $\mathcal{S}_{e}$-directive sequence of a recurrent dendric word $x$ (see Theorem 6 ) the case where its stabilizer is nontrivial, that is, the case where there exists at least one non-trivial substitution $\sigma$ such that $\sigma(x)=x$ ? Can one characterize among substitutive dendric words the dendric words that are fixed points of substitutions? For the two-letter Sturmian case, see, e.g., [6], and the references therein.

The very fact that the family of dendric subshifts encompasses Arnoux-Rauzy subshifts shows the diversity of spectral behaviors of minimal dendric subshifts. Indeed, by [10], Arnoux-Rauzy subshifts can have topological eigenvalues or not; the same holds for measure-theoretic ones. Note that if we focus on dendric subshifts generated by primitive substitutions, it is known that measure-theoretical and topological eigenvalues are the same [24]. One way to tackle spectral questions concerning dendric subshifts is to interpret their $\mathcal{S}_{e}$-adic representation in terms of continued fractions and understand the underlying convergence.

\section{Acknowledgements}

We would like to thank P. Séébold for having pointed out Reference [35], as well as Paulina Cecchi for her careful reading and her valuable comments and suggestions.

\section{References}

[1] N. Aubrun, M.-P. Béal, Tree-shifts of finite type, Theoret. Comput. Sci. 459 (2012), $16-25$.

[2] J. Berstel, C. De Felice, D. Perrin, C. Reutenauer, G. Rindone, Bifix codes and Sturmian words, J. Algebra 369 (2012), 146-202.

[3] V. Berthé, V. Delecroix, Beyond substitutive dynamical systems: S-adic expansions, RIMS Lecture note 'Kokyuroku Bessatu' B46 (2014), 81-123.

[4] V. Berthé, D. Frettlöh, V. Sirvent, Selfdual substitutions in dimension one, European J. of Combinatorics 33 (2012), 981-1000.

[5] V. Berthé, C. Holton, L. Q. Zamboni, Initial powers of Sturmian sequences, Acta Arith. 122 (2006), 315-347.

[6] V. Berthé, H. Ei, S. Ito, H. Rao, On substitution invariant Sturmian words: an application of Rauzy fractals, Theoret. Informatics Appl. 41 (2007), 329-349.

[7] V. Berthé, C. De Felice, F. Dolce, J. Leroy, D. Perrin, C. Reutenauer, G. Rindone, Acyclic, connected and tree sets, Monatsh. Math. 176 (2015), 521-550.

[8] V. Berthé, C. De Felice, F. Dolce, J. Leroy, D. Perrin, C. Reutenauer, G. Rindone, Maximal bifix decoding, Discrete Math. 338 (2015), 725-742.

[9] V. Berthé, C. De Felice, F. Dolce, J. Leroy, D. Perrin, C. Reutenauer, G. Rindone, The finite index basis property, J. Pure Appl. Algebra 219 (2015), 2521-2537. 
[10] J. Cassaigne, S. Ferenczi, A. Messaoudi, Weak mixing and eigenvalues for ArnouxRauzy sequences, Ann. Inst. Fourier (Grenoble) 58 (2008), 1983-2005.

[11] F. M. Dekking, The spectrum of dynamical systems arising from substitutions of constant length, Z. Wahrscheinlichkeitstheorie und Verw. Gebiete, 41 (1977/78), 221239.

[12] V. Diekert, D. Krieger, Some remarks about stabilizers, Theoret. Comput. Sci. 410 (2009), 2935-2946.

[13] F. Dolce, R. Kyriakoglou, J. Leroy, Decidable properties of extension graphs for substitutive languages, 16th Mons Theoretical Computer Science Days, Liège (Belgium).

[14] F. Dolce, D. Perrin, Neutral and tree sets of arbitrary characteristic, Theoretical Computer Science 658 (2017), 159-174.

[15] T. Downarowicz, Survey of odometers and Toeplitz flows, Algebraic and topological dynamics, Contemp. Math. 385 (2005), 7-37, Amer. Math. Soc., Providence, RI.

[16] F. Durand, A characterization of substitutive sequences using return words, Dicrete Math. 179 (1998), 89-101.

[17] F. Durand, Linearly recurrent subshifts have a finite number of non-periodic subshift factors, Ergodic Theory \& Dynam. Systems 20 (2000), 1061-1078. Corrigendum and addendum, Ergodic Theory \& Dynam. Systems 23 (2003), 663-669.

[18] F. Durand, Cobham's theorem for substitutions, J. Eur. Math. Soc. (JEMS) 13 (2011), 1799-1814.

[19] F. Durand, HDOL $\omega$-equivalence and periodicity problems in the primitive case, Unif. Distrib. Theory 7 (2012), 199-215.

[20] F. Durand, M. Rigo, Syndeticity and independent substitutions, Adv. Appl. Math. 42 (2009), 1-22.

[21] F. Durand, J. Leroy and G. Richomme, Do the properties of an S-adic representation determine factor complexity, J. of Integer Sequences 16 (2013), Article 13-2-6.

[22] Fogg, N. Pytheas, Substitutions in dynamics, arithmetics and combinatorics, Springer-Verlag, Berlin, 1794 (2002).

[23] H. Hmili, Non topologically weakly mixing interval exchanges, Discrete Contin. Dyn. Syst. 27 (2010), 1079-1091.

[24] B. Host, Valeurs propres des systèmes dynamiques définis par des substitutions de longueur variable, Ergodic Theory Dynam. Systems 6 (1986) 529-540.

[25] D. Krieger, On stabilizers of infinite words, Theoret. Comput. Sci. 400 (2008), 169181.

[26] J. Leroy, Some improvements of the S-adic conjecture, Adv. in Appl. Math. 48 (2012), 79-98.

[27] J. Leroy, An S-adic characterization of minimal subshifts with first difference of complexity $1 \leq p(n+1)-p(n) \leq 2$, Discrete Math. Theor. Comput. Sci. 16 (2014), $233-286$.

[28] J. Leroy, G. Richomme, A combinatorial proof of $S$-adicity for sequences with linear complexity, Integers 13 (2013), Paper No. A5.

[29] M. Lothaire, Algebraic Combinatorics on Words, Encyclopedia of Mathematics and its Applications, vol. 90, Cambridge University Press, Cambridge, United Kingdom, 2002.

[30] W. Magnus, A. Karrass, D. Solitar, Combinatorial Group Theory, Dover Publications, Inc., Mineola, NY, second edition, 2004.

[31] F. Mignosi, P. Séébold, Morphismes sturmiens et règles de Rauzy, J. Théor. Nombres Bordeaux 5 (1993), 221-233.

[32] B. Mossé, Puissances de mots et reconnaissabilité des points fixes d'une substitution, Theoret. Comput. Sci. 99 (1992), no. 2, 327-334. 
16 Berthé et al.

[33] B. Mossé, Reconnaissabilité des substitutions et complexité des suites automatiques, Bull. Soc. Math. France 124 (1996), no. 2, 329-346.

[34] N. Ormes, Strong orbit realization for minimal homeomorphisms, J. Anal. Math. 71 (1997), 103-133.

[35] J. J. Pansiot, A decidable property of iterated morphisms, in Deussen P. (eds) Theoretical Computer Science, Proc. 5th GI-Conference, Lecture Notes in Computer Science 104 (1981), 152-158.

[36] G. Richomme, Conjugacy and episturmian morphisms, Theoret. Comput. Sci. 302 (2003), 1-34.

[37] G. Richomme, P. Séébold, Completing a combinatorial proof of the rigidity of Sturmian words generated by morphisms, Theoret. Comput. Sci. 428 (2012), 92-97.

[38] P. Séébold, On the conjugation of standard morphisms, Theoret. Comput. Sci. 195 (1998), 91-109.

[39] B. Tan, Z.-X. Wen, Y. Zhang, The structure of invertible substitutions on a three-letter alphabet, Adv. in Appl. Math. 32 (2004), 736-753.

[40] Z.-X. Wen, Z.-Y. Wen, Local isomorphisms of invertible substitutions, C.R. Acad. Sci. Paris, Sér. A 318 (1994), 299-304.

[41] Z.-X. Wen, Y. Zhang, Some remarks on invertible substitutions on three letter alphabet, Chin. Sci. Bulletin 44 (1999) 1755-1760. 